Nota científica

\title{
Registro notable de margay (Leopardus wiedii) en el bosque mesófilo de montaña de Morelos, México
}

\author{
A noteworthy record of margay (Leopardus wiedii) in the cloud forest of Morelos, Mexico \\ Marcelo Aranda ${ }^{\mathrm{a}, *}$ y David Valenzuela-Galván ${ }^{\mathrm{b}}$ \\ ${ }^{a}$ Comisión Nacional de Áreas Naturales Protegidas, Privada Nueva Tabachín 104, Colonia Tlaltenango, 62170, Cuernavaca, Morelos, México \\ ${ }^{\mathrm{b}}$ Centro de Investigación en Biodiversidad y Conservación, Universidad Autónoma del Estado de Morelos. Av. Universidad 1001, Col. Chamilpa, 62209, \\ Cuernavaca, Morelos, México
}

Recibido el 31 de julio de 2015; aceptado el 22 de septiembre de 2015

Disponible en Internet el 12 de noviembre de 2015

\begin{abstract}
Resumen
En junio de 2014 se confirmó la presencia de margay (Leopardus wiedii) en la fracción I del Área de Protección de Flora y Fauna Corredor Biológico Chichinautzin, Morelos, México. El registro se basa en 4 clips de video tomados por una cámara automática ubicada a $2,750 \mathrm{~m}$ de altitud, en bosque mesófilo de montaña. Este registro es la segunda evidencia confirmada de la presencia de poblaciones de margay en el estado de Morelos, así como el primer registro para el Corredor Biológico Chichinautzin, el cual expande el área de distribución del margay en el centro de México, alrededor de $60 \mathrm{~km}$ al norte-noroeste del primer registro para Morelos, en la Reserva de la Biosfera Sierra de Huautla. Derechos Reservados ( 2015 Universidad Nacional Autónoma de México, Instituto de Biología. Este es un artículo de acceso abierto distribuido bajo los términos de la Licencia Creative Commons CC BY-NC-ND 4.0.
\end{abstract}

Palabras clave: Margay; Conservación de felinos silvestres; Extensión de rango

\begin{abstract}
In June2014, we confirmed the presence of margay (Leopardus wiedii) in fraction I of the Área de Protección de Flora y Fauna Corredor Biológico Chichinautzin, Morelos, Mexico. Our record is based in 4 video clips taken by an automatic camera located at $2,750 \mathrm{~m}$ of altitude, with cloud forest. This record is the second confirmed evidence of the presence of margay populations in the state of Morelos, and it is the first record of the species for the Corredor Biológico Chichinautzin, expanding the distribution range of margay into Central Mexico by nearly $60 \mathrm{~km}$ to the north-northwest of the site where it was first recorded for Morelos, at the Sierra de Huautla Biosphere Reserve.

All Rights Reserved (C) 2015 Universidad Nacional Autónoma de México, Instituto de Biología. This is an open access item distributed under the Creative Commons CC License BY-NC-ND 4.0.

Keywords: Margay; Wild felines conservation; Range extension

Entre los mamíferos, los felinos constituyen el grupo más especializado para la caza, con adaptaciones físicas y conductuales para una alimentación estrictamente carnívora (Kitchener, 1991), y en México existen 6 especies (Ceballos y Oliva, 2005): jaguarundi (Herpailurus yagouaroundi), ocelote (Leopardus pardalis), margay (Leopardus wiedii), lince (Lynx rufus), puma (Puma concolor) y jaguar (Panthera onca). El margay

\footnotetext{
* Autor para correspondencia.

Correo electrónico: arandam54@yahoo.com.mx (M. Aranda).

La revisión por pares es responsabilidad de la Universidad Nacional Autó-
} noma de México.

presenta una amplia distribución que va del norte de México al norte de Argentina y Uruguay; ha sido considerado un felino asociado principalmente a bosques conservados, perennifolios y caducifolios, tropicales y subtropicales, desde el nivel del mar y hasta alrededor de los 3,000 m de altitud, aunque en general son pocos los registros por arriba de los 2,000 m (Aranda, 2005; Sunquist y Sunquist, 2002). Se considera que caza tanto en el suelo como en los árboles y que, en general, es poco tolerante a la perturbación. Sin embargo, cada vez hay más evidencia de que puede persistir en sitios alterados, con fragmentos de bosques sin alteración, mezclados con bosques degradados y vegetación secundaria (De Oliveira, 1994, 1998; Reid, 2009;
\end{abstract}


Valenzuela-Galván, de León-Ibarra, Lavalle-Sánchez, OrozcoLugo y Chávez, 2013). En México es un felino considerado en peligro de extinción (Semarnat, 2010); la Unión Internacional para la Conservación de la Naturaleza lo considera una especie casi amenazada, con una tendencia poblacional en decremento (Payan et al., 2008); la Convención sobre el Comercio Internacional de Especies Amenazadas de Flora y Fauna Silvestres (CITES, 2015; http://www.cites.org/esp/app/index.php) ubica al margay en el apéndice I, que incluye a las especies en mayor peligro.

Hasta la década de 1990 no se había documentado la presencia del margay en el estado de Morelos (Álvarez-Castañeda, 1996), pero recientemente en el sureste del estado, en la Reserva de la Biosfera Sierra de Huautla y en bosque tropical caducifolio, se obtuvo el primer registro confirmando su presencia (Valenzuela-Galván et al., 2013). Por otra parte, dado que en el norte de Morelos dominan bosques templados la presencia de algunas especies de origen neotropical como el margay se consideraba poco probable.

En particular, en dicha región de Morelos se ubica el Complejo Corredor Biológico Chichinautzin (Complejo Cobio) constituido por 3 áreas naturales protegidas (ANP) de competencia federal: los parques nacionales Lagunas de Zempoala y El Tepozteco, así como el Área de Protección de Flora y Fauna Corredor Biológico Chichinautzin (Conanp, 2013). Esta última ANP fue decretada en 1988 y tiene una extensión de $37,302.40$ ha, en 2 fracciones: la fracción I con 20,195.68 ha y la fracción II con 17,106.71 ha (Sedue, 1998).

En marzo de 2014 se iniciaron actividades de monitorización de mamíferos en la fracción I del Área de Protección de Flora y Fauna Complejo Cobio, en el extremo noroeste del estado de Morelos, mediante el uso de trampas-cámara (Ltl Acorn 5210A) en varios sitios del bosque mesófilo de montaña. Las cámaras fueron colocadas sobre árboles, a una altura entre 40 y $90 \mathrm{~cm}$ del suelo, programadas para grabar video durante $45 \mathrm{seg}$ a intervalos de 10 min. Entre junio y agosto de 2014, se colocaron varias cámaras en la sección oeste de la fracción I del Complejo Cobio, con un esfuerzo total de muestreo de 228 días-trampa. En una de esas cámaras, establecida a $2,750 \mathrm{~m}$ de altitud, en un punto con coordenadas $19.01^{\circ} \mathrm{O}$ y $99.3^{\circ} \mathrm{N}$, a $3.5 \mathrm{~km}$ al oeste del poblado de Huitzilac, municipio de Huitzilac, Morelos, se obtuvieron 4 clips de video de margay (fig. 1); 2 en junio: el primero, el 2; el segundo, el 28 -ambos en periodo nocturno-; uno el 17 de julio a las 04:54 h; y el último, el 4 de agosto a las 01:31 h. No fue posible confirmar si se trata del mismo individuo, debido a que las tomas no son del mismo costado o cogen al animal de frente o en ángulos que no permiten las comparaciones inequívocas. En la misma cámara se obtuvieron registros de otras especies de mamíferos: Didelphis virginiana, Nasua narica, Odocoileus virginianus y Sciurus aureogaster; pero también de aves: Atlapetes virenticeps, Dendrortyx macroura, Ortalis poliocephala, Peucedra mustaeniatus y Pipilo maculatus.

El bosque mesófilo de montaña tiene una distribución muy limitada en el estado de Morelos, distribuyéndose principalmente en su extremo noroeste donde cubre una superficie menor a 5,000 ha. Es una comunidad densa y húmeda en la que dominan árboles perennifolios y caducifolios, como Quercus
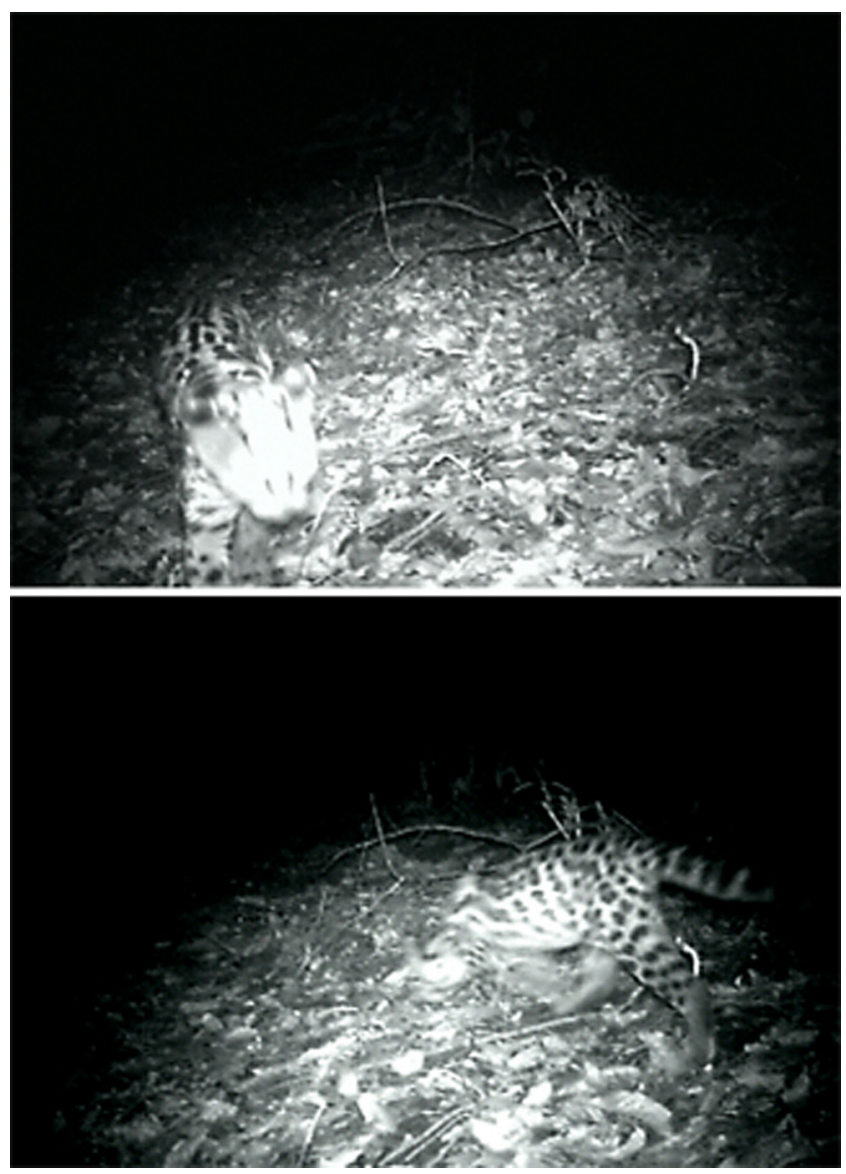

Figura 1. Fotogramas tomados de los 2 clips de video en los que se registró al margay en la fracción I del Área de Protección de Flora y Fauna Corredor Biológico Chichinautzin, en el extremo noroeste del estado de Morelos, México.

laurina, Clethra mexicana, Styrax ramirezii, Cornus disciflora, Meliosma dentata y Arbutus xalapensis (Contreras-MacBeath, Jaramillo y Boyás-Delgado, 2006). El registro de este felino neotropical en esta región de bosques templados se suma al registro reciente de ocelote, en 2013, en el Parque Nacional Lagunas de Zempoala (Aranda, Botello, Martínez-Meyer y Pineda, 2014).

El presente registro de margay se puede considerar notable por varias razones: en la literatura científica no se contaba con ningún indicio que hiciera suponer la existencia de esta especie en la región noroeste del estado de Morelos, aunque comentarios de pobladores locales indicaban cierta probabilidad. Es, además, el segundo registro confirmado de la presencia de margay en el estado. Considerando que se tienen 4 registros en un periodo de casi 3 meses, nos parece menos probable que pueda ser un individuo en dispersión y más probablemente un animal residente, por tanto, nuestros registros podrían indicar la presencia de una población de margay en el área, la cual sería la más céntrica del país y la más cercana a la ciudad de México. El presente registro extiende la distribución de la especie, para el centro de México, en alrededor de $60 \mathrm{~km}$ al norte-noroeste, respecto del registro más cercano en el sur del estado, en la Reserva de la Biosfera Sierra de Huautla (Valenzuela-Galván et al., 2013). Es a su vez uno de los pocos registros de presencia de margay en bosque mesófilo de montaña y el de mayor altitud 
para México. Otros registros de la especie en bosques mesófilos de México se han obtenido en los estados de Jalisco, Oaxaca, Guerrero y San Luis Potosí y van de los 800 a los 2,436 m de altitud (Almazán-Catalán et al., 2013; Aranda, Botello y LópezDe Buen, 2012; Carvajal-Villarreal et al., 2012; Cinta-Magallón, Bonilla-Ruiz, Alarcón y Arroyo-Cabrales, 2012). Los registros previos de mayor altitud para la especie en el país se dieron en Oaxaca en un bosque mesófilo de montaña a 2,436 m de altitud (Cinta-Magallón et al., 2012) y en Guerrero a 2,509 m de altitud, en un bosque de pino (Almazán-Catalán et al., 2013).

El objetivo principal de las ANP es la conservación de la biodiversidad (Conanp, 2013), por tanto, mejorar el conocimiento sobre las especies presentes en ellas es relevante y puede apuntalar la justificación para mantener y reforzar acciones de conservación en un territorio protegido. Con el presente registro de margay, ahora en el Complejo Cobio se tiene la presencia de 4 de las 6 especies de felinos que habitan en México, lo que puede indicar que no obstante su cercanía con grandes desarrollos urbanos como las ciudades de México, Toluca y Cuernavaca y estar sujeto a fuertes presiones ambientales, como la tala, la caza y las actividades agropecuarias, este sistema de ANP ha sido eficaz para la protección de su riqueza biológica. La presencia de carnívoros que están en la cima de la pirámide ecológica indica que la región aún mantiene áreas en buen estado de conservación e integridad ecológica. A la vez, los registros recientes de especies como el margay y el ocelote (ambas especies consideradas en peligro de extinción en la legislación ambiental mexicana; Semarnat, 2010) en esta ANP son relevantes;, destacan la importancia de mantener estas ANP, pero también representan una llamada de alerta para enfatizar acciones para su conservación. Tanto el presente registro de margay como el de ocelote en el sitio de estudio se han dado a altitudes poco comunes para estas especies. Se requerirá mayor investigación y esfuerzo de monitorización para determinar si este es el tipo de respuestas ecológicas que podrían estar asociadas a las modificaciones ambientales derivadas del cambio climático global (Parmesan, 2006). Desde luego, también es claro que para muchos sitios y condiciones el esfuerzo de muestreo ha sido insuficiente y el incremento en el trabajo de campo en dichos escenarios aportará nuevos registros. Sin embargo, para el caso de Lagunas de Zempoala, al menos hay un antecedente que implicó un buen esfuerzo de muestreo sin haber logrado el registro de margay (o de ocelote; Ramírez-Pulido, 1969), lo que apoya parcialmente el que la presencia de estos felinos en la zona puede ser relativamente reciente.

\section{Referencias}

Almazán-Catalán, J. A., Sánchez-Hernández, C., Ruiz-Gutiérrez, F., RomeroAlmaraz, M. L., Taboada-Salgado, A., Beltrán-Sánchez, E., et al. (2013). Registros adicionales de felinos del estado de Guerrero. Revista Mexicana de Biodiversidad, 84, 347-359.

Álvarez-Castañeda, S. T. (1996). Los mamíferos del estado de Morelos. La Paz: Centro de Investigaciones Biológicas del Noroeste, S.C.

Aranda, M. (2005). Leopardus wiedii (Schinz, 1821). En: G. Ceballos y G. Oliva (Coords.), Los mamíferos silvestres de México (pp. 361-362). México, D.F.:
Comisión Nacional para el Conocimiento y Uso de la Biodiversidad/Fondo de Cultura Económica.

Aranda, M., Botello, F. y López-De Buen, L. (2012). Diversidad y datos reproductivos de mamíferos medianos y grandes en el bosque mesófilo de montaña de la Reserva de la Biosfera Sierra de Manantlán, Jalisco-Colima, México. Revista Mexicana de Biodiversidad, 83, 778-784.

Aranda, M., Botello, F., Martínez-Meyer, E. y Pineda, A. (2014). Primer registro de ocelote (Leopardus pardalis) en el Parque Nacional Lagunas de Zempoala, Estado de México y Morelos, México. Revista Mexicana de Biodiversidad, 85, 1300-1302.

Carvajal-Villarreal, S., Caso, A., Downey, P., Moreno, A., Tewes, M. E. y Grassman, L. I., Jr. (2012). Spatial patterns of the margay (Leopardus wiedii; Felidae, Carnivora) at "El Cielo" Biosphere Reserve, Tamaulipas, Mexico. Mammalia, 76, 237-244.

Ceballos, G. y Oliva, G. (Coords.). (2005). Los mamíferos silvestres de México. México, D.F.: Comisión Nacional para el Conocimiento y Uso de la Biodiversidad/Fondo de Cultura Económica.

Cinta-Magallón, C., Bonilla-Ruiz, C., Alarcón, R. y Arroyo-Cabrales, J. (2012). Dos nuevos registros de margay (Leopardus wiedii) en Oaxaca, México, con datos sobre hábitos alimentarios. Research Journal of the Costa Rican Distance Education University, 41, 33-40.

CITES (Convención sobre el Comercio Internacional de Especies Amenazadas de Fauna y Flora Silvestres). (2015). Recuperado el 14 de septiembre de 2015 de: http://www.cites.org/esp/app/index.php

Conanp (Comisión Nacional de Áreas Naturales Protegidas). (2013). Recuperado el 5 de noviembre de 2013 de: www.conanp.gob.mx.

Contreras-MacBeath, T., Jaramillo, F. y Boyás-Delgado, J.C. (Coords.). (2006). La diversidad biológica en Morelos. México, D.F.: Comisión Nacional para el Conocimiento y Uso de la Biodiversidad/Universidad Autónoma del Estado de Morelos.

De Oliveira, T. G. (1994). Neotropicalcats: ecology and conservation. São Luís: Editora da Universidade Federal do Maranhão.

De Oliveira, T. G. (1998). Leopardus wiedii. Mammalian Species, 579 $1-6$.

Kitchener, A. (1991). The natural history of the wild cats. Ithaca: Comstock Publishing Associates.

Parmesan, C. (2006). Ecological and evolutionary responses to recent climate change. AnnualReview of Ecology, Evolution and Systematics, 37, 637-669

Payan, E., Elzirik, E., De Oliveira, T., Leite-Pilman, R., Kelly, M. y Valderrama, C. (2008). Leopardus wiedii. The IUCN Red List of Threatened Species. Version 2014.2. Recuperado el 20 de agosto de 2014 de: www.iucnredlist.org

Ramírez-Pulido, J. (1969). Contribución al estudio de los mamíferos del Parque Nacional Lagunas de Zempoala, Morelos, México. Anales del Instituto de Biología, Universidad Nacional Autónoma de México, serie Zoología, 40, 253-290.

Reid, F. A. (2009). A field guide to the mammals of Central America and Southeast Mexico, $\left(2 .^{a}\right.$ ed.). Oxford: Oxford University Press.

Sedue (Secretaría de Desarrollo Urbano y Ecología). (1998). Decreto por el que se declara el Área de Protección de Flora y Fauna Silvestre, ubicada en los municipios de Huitzilac, Cuernavaca, Tepoztlán, Jiutepec, Tlalnepantla, Yautepec, Tlayacapan y Totolapan, Morelos. Diario Oficial de la Federación. 30 de noviembre de 1988. México.

Semarnat (Secretaría de Medio Ambiente y Recursos Naturales). (2010). Norma Oficial Mexicana NOM-059-SEMARNAT-2010, Protección ambientalEspecies nativas de México de flora y fauna silvestres-Categorías de riesgo y especificaciones para su inclusión, exclusión o cambio-Lista de especies en riesgo. Diario Oficial de la Federación. 30 de diciembre de 2010, Segunda Sección. México.

Sunquist, M. y Sunquist, F. (2002). Wildcats of the world. Chicago: The University of Chicago Press.

Valenzuela-Galván, D., de León-Ibarra, A., Lavalle-Sánchez, A., Orozco-Lugo, L. y Chávez, C. (2013). The margay Leopardus wiedii and bobcat Lynx rufus from the dry forest of Southern Morelos, Mexico. The Southwestern Naturalist, 58, 118-120. 